\title{
Key Factors to Address the Issue of Global Energy and Environment Crisis
}

\author{
Zhiqiang Liu*
}

DOI: $10.30919 / \mathrm{esmm} 5 \mathrm{f} 225$

Energy and environment are critical important for our sustainable development. Novel materials and nanomanufacturing techniques provide new opportunities for this important research area. This issue has nine interesting papers dedicated to thermoelectrics, alkali-activated materials (AAMs), nano-materials, and perovskite quantum dots.

With growing concerns for greenhouse gas emissions, AAMs have received enormous attention due to the benefit of low carbon footprint. Wengui Li et al. (DOI: 10.30919/esmm5f204) presents a critical review on the durability performance of alkali-activated system. It will provide guidelines for the research community as well as to the stakeholders of AAMs industries who seek sustainability in their products.

Nitride materials have been widely used in light- emitting devices for decades. However, highly p-doping is still a big challenge, with hinds the further development of nitride-based devices. Liu et al. (DOI: 10.30919/esmm5f209) clarified the underlying physics and the acceptor ionization process in the $\mathrm{In}-\mathrm{Mg}$ co-doping $\mathrm{GaN}$, which is widely applicable for other co-doping nitride systems. The results will advance the current understanding of p-doping challenge and help to further address the issue of p-doping in wide-bandgap materials, especially from the viewpoint of band-structure engineering.

Thermoelectrics is one of the most promising solutions to address the global energy crisis. Shuang Tang (DOI: 10.30919/esmm5f213) developed an efficient thermoelectric indicator based on band structure information to search for the most promising candidates from various band structure databases. This work provides new physical insights for searching and improving thermoelectric materials, and will stimulate more novel work in this field following the advancement in firstprinciples calculations to predict materials properties. It will provide important guidance for researchers to select and better engineer new materials.

In recent years, as significant efforts have been emphasized on the exploitation of novel polymers with enhanced functionality. Yue Chen et al. (DOI: 10.30919/esmm5f214) developed a self-healing supramolecular polymer composite (LP-GO), which was designed and prepared via incorporation of graphene oxide $(\mathrm{GO})$ to hyperbranched polymer. The simplicity of synthesis and the availability from renewable resources will ensure broad applications of these composites.

The recycling of photocatalysts is in great demand. The microsized magnetic $\mathrm{Fe}_{3} \mathrm{O}_{4}$ powders were successfully encapsulated by carbon-doped anatase titanium by Baolai Wang et al.(DOI: 10.30919/esmm5f215). The present work could provide a new route to improve the photocatalysis efficiency and the recycles of photocatalysts.

Research and Development Center for Solid State Lighting, Institute of Semiconductors, Chinese Academy of Sciences, Beijing 100083, China

*E-mail: lzq@semi.ac.cn
In order to increase the gas barrier performance of food packaging PET films, YiFan Chen et al. (DOI: 10.30919/esmm5f216) used the intercalated sericite as a barrier material in the functional silicone resin coatings. The silicone resin modified by intercalated sericite successfully prevented the direct penetration of gas molecules and exhibited high barrier performance.

$\mathrm{PbS}$ materials have important research value in basic theory, high application value in many fields, such as optoelectronic devices and

biomedical imaging. Rui Li et al. (DOI: 10.30919/esmm5f217) systematically analyzed the effect of $\mathrm{Cu}$ doping on the structural, morphological, optical and electrical properties of $\mathrm{PbS}$ nanofilms. They identified the optimal concentration of $\mathrm{Cu}$ doping for sunlight absorbing layers and conductive layers, respectively.

Polyolefins, including polyethylene (PE) and polypropylene (PP), are most used important materials employed in commercial commodities, industrial applications and agricultural applications. Shiyun Li et al. (DOI: 10.30919/esmm5f218 ) reported a successful way to synthesis polydivinylbenzene block hyperbranched polyethylene copolymers via in-situ copolymerization by the Atom Transfer Radical Polymerization method.

Halide perovskite quantum dots (QDs) have been considered to be an outstanding optoelectronic material. Wenzhi Wang et al. (DOI: 10.30919/esmm5f219) report a simple silica $\left(\mathrm{SiO}_{2}\right)$-coating process to solve the problem of poor stability without affecting their optical properties. This material and method provide a novel platform and pave the way for the application of perovskite quantum dots in light-emitting device applications.

In summary, new technologies of nanocomposites and nanostructures are key factors to address the issue of global energy and environment crisis. We anticipate more papers and new efforts in coming issues.

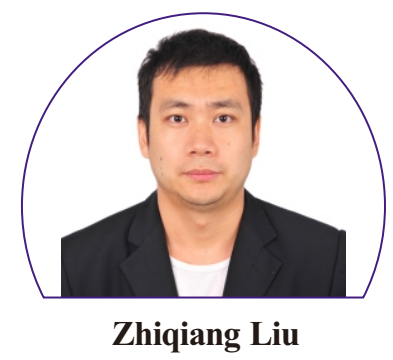

Zhiqiang Liu is currently based Institute of Semiconductors, Chinese Academy of Sciences (IOS, CAS). His research expertise is in building interdisciplinary teams to use compound semiconductor materials and devices for applications in the areas of nitride materials and Light Emitting devices. 